\title{
Supply Estimation Using Coevolutionary Genetic Algorithms in the Spanish Electrical Market
}

\author{
ENRIQUE A. DE LA CAL MARÍN AND LUCIANO SÁNCHEZ RAMOS \\ Computer Science Department, University of Oviedo, Asturias, Spain
}

\begin{abstract}
The price of electrical energy in Spain has not been regulated by the government since 1998, but determined by the supply from the generators in a competitive market, the so-called "electrical pool". A genetic method for analyzing data from this new market is presented in this paper. The eventual objective is to determine the individual supply curves of the competitive agents. Adopting the point of view of the game theory, different genetic algorithm configurations using coevolutionary and non-coevolutionary strategies combined with scalar and multi-objective fitness are compared. The results obtained are the first step toward solving the induction of the optimal individual strategies into the Spanish electrical market from data in terms of perfect oligopolistic behavior.
\end{abstract}

Keywords: intelligent data analysis, coevolutionary genetic algorithms, evolutionary economic models

\section{Introduction}

The cost of production of electrical energy depends on the type of generator: one MW produced by a nuclear plant costs less than one MW produced by a thermal plant. The most economical plants are in operation most of the time while the more expensive ones connect to the electrical system only when the former cannot cover demand. Consequently, the cost of energy is higher during peak consumption and lower during the hours of less demand, for instance at night.

Many other factors intervene in the selection of the power plants that connect to the system at any given time, such as geographic location, which affects the loss of energy in the transport lines, or the precipitation rate with regard to the operation of hydraulic plants. In spite of this, the basic principle that "the cheaper power plants connect first" explains the majority of the fluctuations in the cost of energy.

The relationship between the cost of production and the selling price is not direct. Production cost determines price in a regulated market, such as the one that existed in Spain before 1998 and continues to exist in other European Community countries. This is not so in a competitive market. Before 1998, prices in Spain were fixed by a public agency that was also in charge of elaborating a list of the power plants that should connect at any given time. This list was calculated by means of numeric optimization algorithms, which minimized the global cost of the production necessary to cover domestic demand.

In the modern model, based on free competition among the different companies [1], the Market Operator (MO), a neutral agent appointed by the State to regulate competition, calculates the energy prices for every hour, starting from the supply of the generators and the demand of the consumers. The procedure by which production is planned again is based on the principle that "the cheapest power plants connect first". In this case, however, "cheapest" does not mean "low cost", but "low selling price", because each agent is free to choose the price it wants to charge for its power. It is interesting to note that the law stipulates that all power plants are to receive the same payment for each MW of energy sold, as occurred in the non-competitive model, and not the payment they asked for in their strategy. The second principle of the competitive market is "the most costly power plant connected marks the price".

This way of assigning production poses problems difficult for the planning departments of the generating companies to resolve. On the one hand, management of the power plants would be tempted to offer energy 
at zero cost, such that that power plant would always be selected (and paid according to the price marked by competition). However, this strategy, if applied by a considerable fraction of the companies, would cause a global decrease in prices in the short run, to the detriment of all the generators. On the other hand, the opposite temptation, to agree on prices, much more efficient in economical terms, is prohibited by law. The only legal way to maximize the profit of a company is to accurately predict demand as well as the price that competition will offer. This way, the agent can adjust its own price so that it is slightly under market price and thus be assigned the greatest possible amount of production at a high price.

Demand can be predicted using simple statistical techniques, but not supply. In reality, not only is it difficult to predict future supply, but also to know past supply: the MO guarantees that this information will remain confidential until a certain amount of time has transpired. In order to predict supply, the first step is to elaborate an intelligent data analysis tool that is capable of estimating the past supply of the agents based on the energy prices and the hours of consumption. That is the main objective of this work.

\subsection{Formulation of the Problem in Terms of the Game Theory}

Given that preliminary data are insufficient to carry out a statistical analysis, it is necessary to make conjectures regarding the results. This work assumes that the agents are intelligent and that the market is fair, such that the unit profits (euros/MW) are approximately the same for all the competitors.

With this hypothesis, if we know the cost of production of the agents (and we can estimate that using data prior to 1998), it is possible to simplify market operation and abstract it to a game, which can be explained as follows. Let us assume that a certain amount of energy is to be bought from several generators. None of them is capable of supplying the total amount and the amount supplied by all of them exceeds the needs.

Each player (one of the generators) gives a referee (the MO) a closed envelope with its sales strategy. It consists of a pair "quantity supplied-price demanded per unit". The referee opens the envelopes, arranges the strategies and chooses the cheapest ones until demand is covered. Each player selected is then paid for the amount it sells at the price of the most expensive strategy that was accepted. Each player receives the difference between the price paid and their unit cost, multiplied by the energy units sold.

The actual number of players is several hundred (one player per electrical power plant). To simplify calculations, we group the price-quantity pairs of all the power plants belonging to the same company into a single total quantity produced-unit price curve. In this way, we reduce several hundred strategies to four aggregate supply curves (there are four large electrical companies in Spain). The same is done with costs: each of the four participants in the simplified game will have a curve that relates the negotiated MW with their production cost. The mechanism of this new game is a bit more complex: each player gives the referee an aggregate supply curve. The referee adds up all the curves and intersects the results with a demand curve. The cross point determines the market price. Given the price and the supply curves furnished by the agents, the revenue of each player is calculated. Finally, the net profit of each player is calculated using the difference between the income received and the value of its cost curve at the point corresponding to the amount negotiated.

\subsection{Genetic Formulation}

In this work, we want to reconstruct the aggregate supply curves from a file that contains the price values and the amounts sold in various game repetitions. To do this, a coevolutionary genetic algorithm is used.

Briefly, a genetic algorithm works as follows: first, we define as many populations of strategies as players. To score a strategy, we will simulate a game, making this strategy compete with the best strategy from each of the other players. The strategy will receive a high score if it fulfils these two criteria:

- That the price obtained in the simulation is similar to the price in the real game.

- That the unit profits obtained by each player are similar.

Observe that genetic algorithms have been applied to solve economic problems similar to the one considered int this paper (see [2-7]) and a market model that shares some of the characteristics of this one has been related to a coevolutionary genetic programmingbased model above [8]. Unfortunately, in our opinion, none of these approaches can be extended to solve the precise problem we pose here. 


\subsection{Summary}

The remainder of this paper is arranged as follows: In Section 2, our methodology is described. In Section 3 , a simple problem is solved to illustrate the use of the method proposed here. A self-criticism of the proposed methodology is made in Section 4. In Section 5, co-evolutionary, evolutionary and classical methods, applied to a semi-synthetic problem, are compared. The paper finishes with concluding remarks and future work, and with an appendix containing numerical data from experiments depicted in Section 5.

\section{Proposed Methodology}

The algorithm studied in this work serves to obtain the aggregate supply curves of the companies competing in the market using the historical information from the results of several previous markets.

In the introduction, it was mentioned that these aggregate supply curves provide the price at which a company is prepared to sell its energy, depending on the amount bought. Each supply curve represents a market strategy and the companies elaborate them based on their assumptions with regard to the evolution of demand and the strategies of the other competitors.

\subsection{Definition of a Supply Curve}

Each preliminary datum is a pair formed by two numbers: the total amount of energy produced in Spain during a certain hour and the price of energy at that hour. This pair of values corresponds to a point on the curve obtained by adding up all the supply curves furnished by the players in the market corresponding to that hour, as explained in the introduction. Unfortunately, unless we assume that an agent uses the same curve several times at different hours, the preliminary data do not contain sufficient information to reconstruct the supply curves.

An extreme case consists in assuming that the agents always use the same curve in all the games. This hypothesis is overly simplified. The behavior expected from an agent consists in its demanding higher prices when the forecasted demand is higher: this way, it maximizes its income without risking its offer being rejected. We indeed know that the supply curves that a company uses depend on specific factors. Strategic Planning Departments take into consideration the day of the week, the hour of the day, the season, the weather forecast (rain, temperature) and some other indicators before posting prices to the Market Operator. Our analysis would be very imprecise if we did not consider some of these factors. Following our own experience, three features should be considered: the hour (which is related to the amount of energy negotiated, depending on labor hours and daylight), the day of the week (the dependence between labor hours and demand changes on weekends and holidays) and the season (electrical cooling or heating, affects both previous dependencies).

Given this information, we decided to stay in an intermediate position between (a) assuming that the supply curve is always the same for each agent, and (b) assuming a different curve for every market. Since (a) is too imprecise and (b) is intractable, in this work we will allow each agent to select its curve from a restricted set of choices, depending on the values of the features mentioned before. In other words, a strategy comprises:

- a rule-based classification system, that produces a segmentation of the market points into a certain number of classes depending on hour, day of the week and type of day, and

- as many supply curves as market segments.

That is, each individual is a set of rules whose antecedents are assertions with regard to market characteristics and whose consequents are the supply curves that the player can use. We shall call these consequents "prototype strategies".

The simplest representation of a prototype strategy is a straight line. Linear models can approximate the behavior of a competitive electrical market in the neighborhood of its equilibrium point. Unfortunately, in spite of this kind of simplification, which is valid for studying the response of the market under small changes, it is not accurate enough to estimate complete supply curves of the agents, which are highly non linear. We have decided to use piecewise linear supply curves instead (see Fig. 1). The number of their segments will be a compromise between the accuracy of the model and the amount of available data (three segments in most of the experiments in this paper.)

\subsection{Genetic Representation of Individuals}

We will solve this problem either with regular genetic algorithms or with coevolutionary genetic algorithms, to compare their relative performances. 

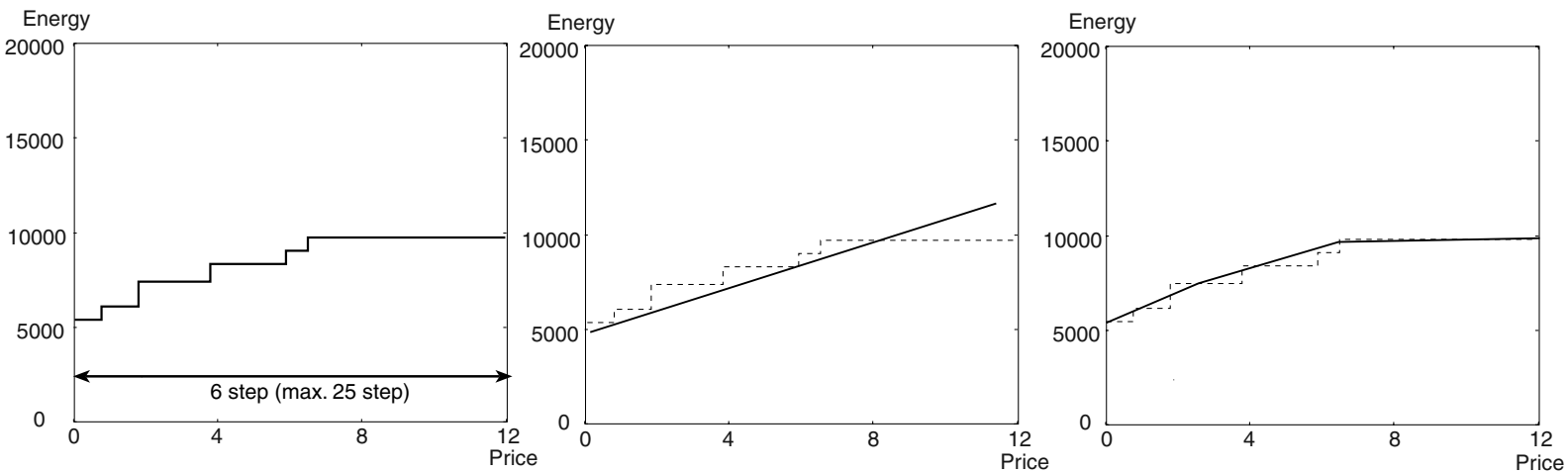

Figure 1. Actual (left), linear (center) and polygonal supply curves (right). Representation by a polygonal line is closer to reality than the linear supply and does not depend on an excessive number of parameters

Each individual in the coevolutionary approach $[9$, 10] codifies a possible set of strategies (i.e., a rule-based classifier system and a set of prototype strategies) of one of the agents; we will keep as many populations of individuals as agents exist. Fitness is not assigned to an individual but to a combination of individuals extracted from all populations [11, 12]. Conversely, individuals in the regular (non coevolutionary) approach are sets that contain one set of strategies for each agent involved in the market, therefore they can be directly assigned a fitness value. The differences in representation, fitness and genetic operators between both ap- proaches are discussed in more detail in the sections that follow.

An individual in the coevolutionary approach will be codified with a chain of numbers. This chain comprises two real numbers to define every segment in a prototype, plus a list containing the numerical parameters on which the linguistic terms in the antecedents of the classifier depend.

To clarify the codification of an individual, let us consider the example in Fig. 2. Let us suppose we have two input variables, called "hour of the day" and "type of day." The first variable can take values from 0 to

\section{Genetic representation}

\section{Graphical representation}
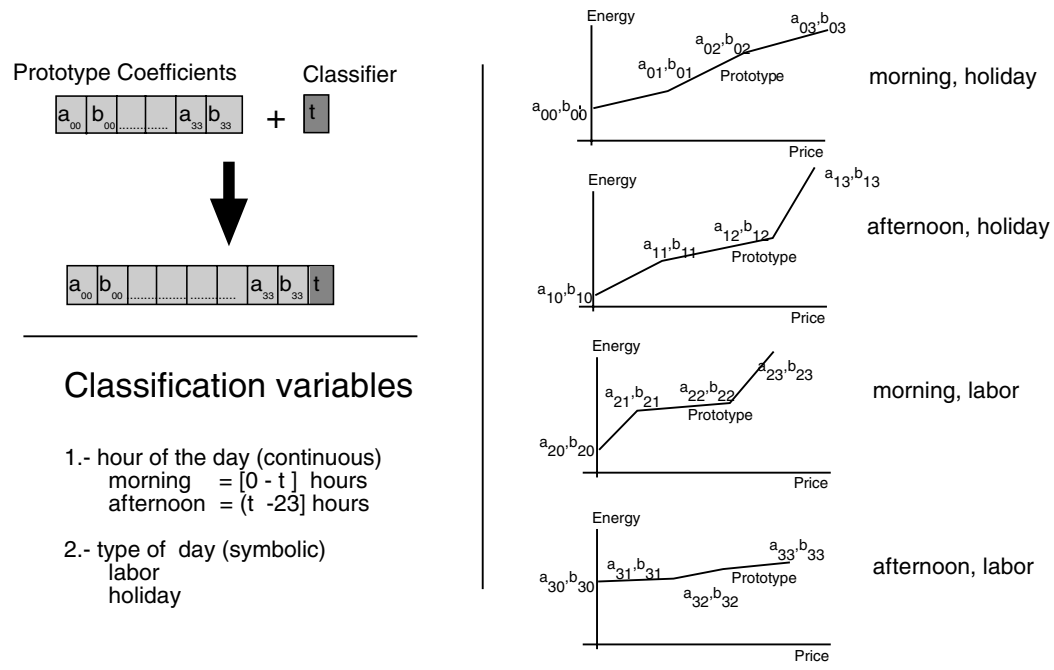

Figure 2. Polygonal supply curve comprising three segments and a classifier with two variables that segments the markets into 4 clusters; (top left) genetic representation, (bottom left) classifier variable values, (right) graphical representation. 
23, and the second one can take two linguistic values, "labor" and "holiday". The antecedents of the rules that compound the strategy must span all values of the input variables; we discretize all continuous variables into linguistic terms first, and then enumerate all possibilities. Let us call "morning" the hours before a time called $t$, and "afternoon" the hours after $t$. The complete strategy will be

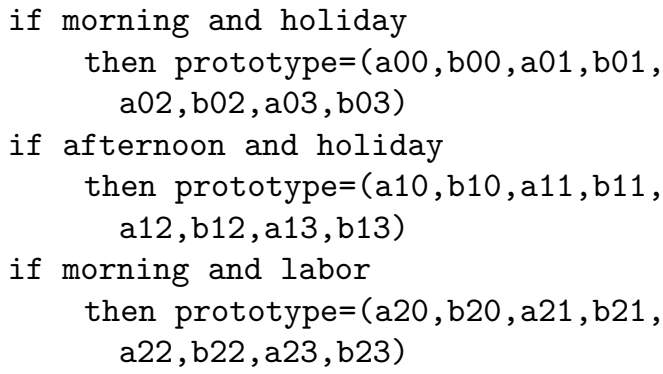

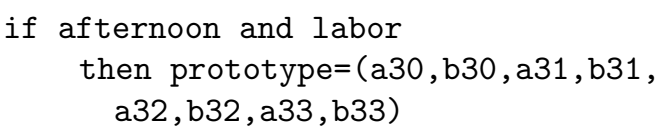

and it can be codified by a chain of 33 numbers. Since each prototype strategy depends on eight values, we need $4 \times 8$ parameters to define all consequents and one more number to define the value of $t$. The antecedents need not to be codified, because they are implicit in the sorting of the rules.

In general, a strategy depending on $n$ input variables, taking $n_{i}$ different values each, will be codified by a chain of $8 \prod_{i=1}^{n} n_{i}+C$, where $C$ is the number of parameters defining the classifier.

The coevolutionary approach uses the number of populations equal to the number of firms participating the market (four, in our case.). Each individual represents the supply strategy for a firm (see Fig. 3

\section{Coevolutionary Approach}
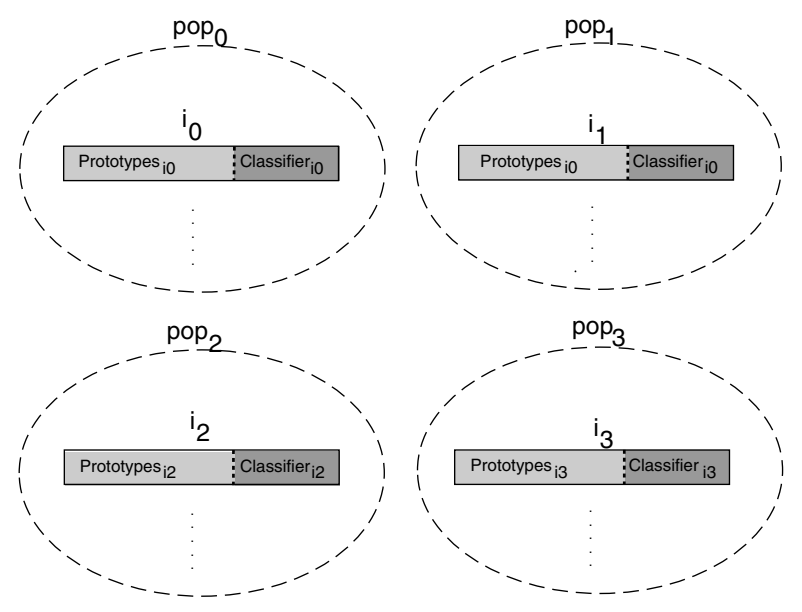

Non-Coevolutionary/Regular Approach

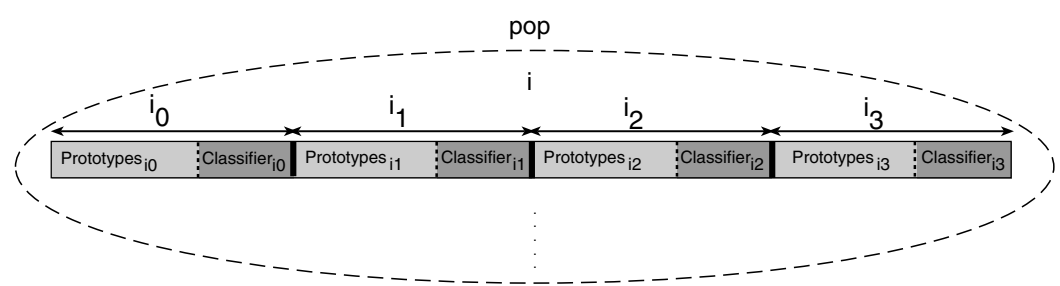

Figure 3. Genetic representation for coevolutionary approach individuals (top), and an aggregate individual (bottom) valid for the noncoevolutionary approach. 
top.) Individuals in the non-coevolutionary approach are formed by concatenating as many individuals of the former type as agents exist (see Fig. 3 bottom.) Observe that the segmentation of the markets is different for every agent, thus they do not share the parameters of the classifier.

\subsection{Genetic Operators}

Individuals in the coevolutionary approach are represented by chains of real numbers, thus there is no need to define custom genetic operators. However, the relative sizes of the subchain codifying the consequents and the subchain codifying the list of parameters of the classifier are very different. We have opted to let only one of these parts be modified in every genetic operation, thus we can manually balance the evolution of both and speed up the evolution of the classifier part. This is the only difference between our operators and the standard versions of uniform arithmetic crossover and mutation [13, 14]. Observe that the offspring is always valid, because:

- The consequents produced by the either the mutation or the crossover operators are weighted sums of monotonic functions, which are also monotonic functions.

- The classifier arising from crossover or mutation is checked, and repaired if needed, so that all parameters defining it are constrained to their respective ranges.

When two individuals are to be crossed, a coin is tossed to decide whether we select from each of them (a) the subchain codifying the classifier definition or (b) one subchain that codifies the definition of one of the prototypes (the consequent of a single rule is modified after applying crossover.) The selected subchains are recombined by means of standard arithmetic crossover, but the remaining part of the individual remains untouched (see Fig. 4 top).

The mutation operator is defined as the crossing of an individual with another one, generated at random. Both

\section{Coevolutionary Approach Crossover}

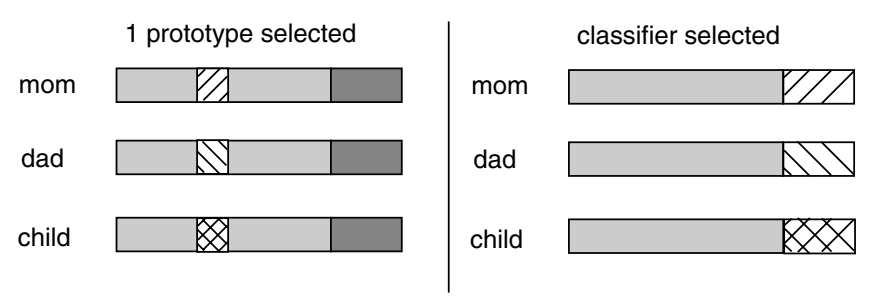

Non-Coevolutionary/Regular Approach Crossover

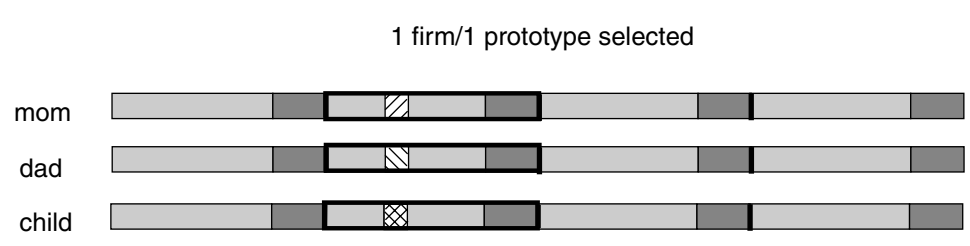

1 firm/classifier selected

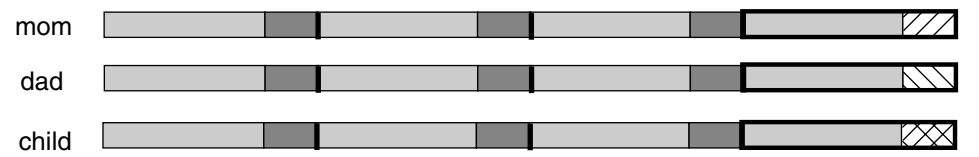

Figure 4. Crossover operations in coevolutionary (top) and evolutionary (bottom) representations. Evolutionary crossover consists of selecting one agent at random and performing the coevolutionary crossover over the corresponding parts. 
recombination and mutation are immediately extended to the canonical case (non-coevolutionary): the same strategy is selected from both parents (i.e., crossover affects subchains with the same relative position inside the individual) and the preceding operations are applied to the selected subchain (see Fig. 4 bottom).

\subsection{Fitness Function}

We have mentioned that we needed to assume that the unitary profits of all firms are the same in order to obtain a good model. Otherwise, we would not be able to determine the market share of every firm, only an aggregation of the supply curves of all firms that could be broken down in many different ways.

This decision implies that we need to rank strategies according to two different criteria: a strategy is good when, after being combined with the strategies of the remaining players, (a) either the price and the quantity sold are near the actual price or quantity and (b) similar unitary profits are achieved by all players -where "unitary profit" is defined as the difference between cost and income, divided by the number of MWs sold. The first goal measures how the curves fit real data, and the second one measures the degree of fulfillment of the restriction "all unitary profits are the same." We will use the square error to quantify the first objective, and the mean of the variances of the unitary profits to quantify the second one.

Different methods exist for mapping multi-objective fitness into scalar fitness [15]. We have studied the weighted average of values (a) and (b), but, according to our experiments (see Section A.2), there is a significant improvement if we use a multi-objective approach instead [16-19].

The same fitness function was applied to both the coevolutionary process (as many populations as companies) and the regular process (one population). Note that in the coevolutionary approach, the fitness of an individual is calculated making it compete with individuals selected at random from other populations, while in the evolutionary approach, an individual is a set of compound strategies from all companies, thus this selection is not necessary.

\section{Demonstration Case}

A simple problem is presented here for the sake of illustrating the basic aspects of the proposed method-
Table 1. Market points in the demonstration case.

\begin{tabular}{lcccccccccr}
\hline Price & 12.5 & 15 & 17.5 & 20 & 22.5 & 25 & 27.5 & 30 & 32.5 & 35 \\
Quantity & 75 & 80 & 85 & 90 & 95 & 100 & 105 & 110 & 115 & 120 \\
\hline
\end{tabular}

ology. This example models 10 repetitions of a game in which we know each player always uses the same supply curve, thus we do not need the classifier. Supplies are straight lines, each depending on two parameters.

The inputs for this problem are:

1. The cost functions. $q$ is the quantity of energy produced, $C_{0}$ and $C_{1}$ are the prices demanded:

$$
\begin{aligned}
& C_{0}(q)=8 q+12 \\
& C_{1}(q)=8.5 q+10
\end{aligned}
$$

2. The market scenario, a series of 10 demand functions $\left(D_{m}\right)$ with the same elasticity (i.e., the same steepness):

$$
D_{m}(p)=-2 p+(100+10 m), \quad \text { for } m \text { in } 0 \ldots 9
$$

3. The set of market points in Table 1, (price $_{i}$, quantity $_{i}$ ). They were generated from the intersections of the demand functions $\left(D_{i}\right)$ and the aggregate strategy.

The original strategies of the agents (those we want to recover) are:

$$
\begin{aligned}
& q_{0}^{\text {orig }}(p)=0.10 p+16.60 \\
& q_{1}^{\text {orig }}(p)=1.89 p+33.55
\end{aligned}
$$

therefore, the intersections of $q_{0}^{\text {orig }}+q_{1}^{\text {orig }}$ and the preceding demand functions produce Table 1 .

The algorithm is fed with Table 1 , functions $D_{0}$ to $D_{9}$ and the cost functions $C_{0}$ and $C_{1}$. It produces approximations $q_{0}, q_{1}$ to the actual strategies $q_{0}^{\text {orig }}$ and $q_{1}^{\text {orig }}$. Individuals in the coevolutionary genetic populations are chains of two numbers (coefficients defining $q_{0}$ or $\left.q_{1}\right)$. For the sake of clarity, let us evaluate the combination of two individuals generated at random, $(0.2,15.0)$ and $(2.0,10.0)$ which correspond to the pair of strategies:

$$
\begin{aligned}
& q_{0}(p)=0.2 p+15.0 \\
& q_{1}(p)=2.0 p+10.0
\end{aligned}
$$


Table 2. Price of energy and total number of units sold for the pair of offers defined in Section 3.

\begin{tabular}{lrrrrrrrrrr}
\hline Price & 17.86 & 20.24 & 22.62 & 25 & 27.38 & 29.76 & 32.14 & 34.52 & 36.90 & 39.29 \\
Quantity & 64.29 & 69.52 & 74.76 & 80 & 85.24 & 90.48 & 95.71 & 100.95 & 106.19 & 111.43 \\
\hline
\end{tabular}

Table 3. Number of units sold by each player.

$\begin{array}{lllllllllll}q_{0} & 18.57 & 19.05 & 19.52 & 20 & 20.48 & 20.95 & 21.43 & 21.90 & 22.38 & 22.86\end{array}$ $\begin{array}{lllllllllll}q_{1} & 45.71 & 50.48 & 55.24 & 60 & 64.76 & 69.52 & 74.29 & 79.05 & 83.81 & 88.57\end{array}$

One part of the fitness depends on the difference between the energy that was actually sold and the energy that would be sold if the strategies being evaluated were issued. We must first calculate the estimated prices for each market. These prices are graphically determined by the cut of $q_{0}+q_{1}$ with each demand $D_{m}$. The results are in Table 2. The squared difference between the quantities in this table and those in Table 1 is 93.5 . The other term in the fitness is the variance of the unitary benefits in all markets. Given the expressions of $q_{0}$ and $q_{1}$, the amount of energy each player sells in each market is contained in Table 3. Unitary profit is calculated by dividing the difference between income $p \cdot q(p)$ and cost $C(q(p))$ into the number $q$ of sold units. The variance of all these values is 0.014 . This is the second field in the fitness value.

Once the models of representation and the combinations of the individuals with the demand curves are defined, a coevolutionary genetic algorithm is applied to obtain an estimate of the original individual strategies. Estimated individual strategies were obtained after running the algorithm with two subpopulations with the size of 400, multicriteria fitness, 200 generations, tournament selection (size 4) and linear descending crossover probability, from 100 to $0 \%$ (this decision is experimentally justified in Appendix A.6). The output of our method is:

$$
\begin{aligned}
& q_{0}(p)=0.10 p+16.70 \\
& q_{1}(p)=1.90 p+33.29
\end{aligned}
$$

\section{Self-Criticism: Non Coevolutionary Analysis of the Data}

One may wonder whether this method offers significant advantages over simpler techniques. Let us suppose all agents share the same classifier, i.e., the an- tecedents of all rules are the same for all agents (for instance, let us suppose all players agree in the meaning of 'morning' and 'afternoon' in the previous example.)

In this case, we can think of applying cluster analysis to the input data (the pairs price-quantity) to estimate the antecedents of the classifier or, in other words, to do a segmentation of the different markets, according to their properties. Then, the input data can be divided into different subsets (market segments), and an aggregate supply curve can be fitted into each market segment by non linear regression (see Fig. 5). The share of all agents is obtained with the help of the unitary profit assumption.

To assess this method, we will set up a second algorithm, in which k-means is used to cluster the points and a genetic algorithm is used to fit a piecewise linear curve to each cluster. We will call this approach " $K$-means Genetic Model" (KGM). From now on, the methods proposed here in their canonical and coevolutionary versions will be called "Genetic Model" (GM) and "Coevolutionary Genetic Model" (CGM), respectively. We will show, by means of numerical simulation of a simplified real problem, that the adjustment of CGM improves that of KGM and GM.

\section{Practical Application: A Simplified Real Problem}

This section describes the application of this method to a semi-synthetic problem. This problem was designed to reproduce current scenarios in the Spanish electrical market, while being originated by theoretical supply curves, thus we can quantify the accuracy of GM, CGM and KGM methods.

The exposition is organized as follows:

1. A description of the semi-synthetic problem is made.

2. The experimental framework (decisions about the genetic process) is defined.

3. KGM, GM and CGM methods are compared.

4. The results are discussed and the practical application of the models studied. 


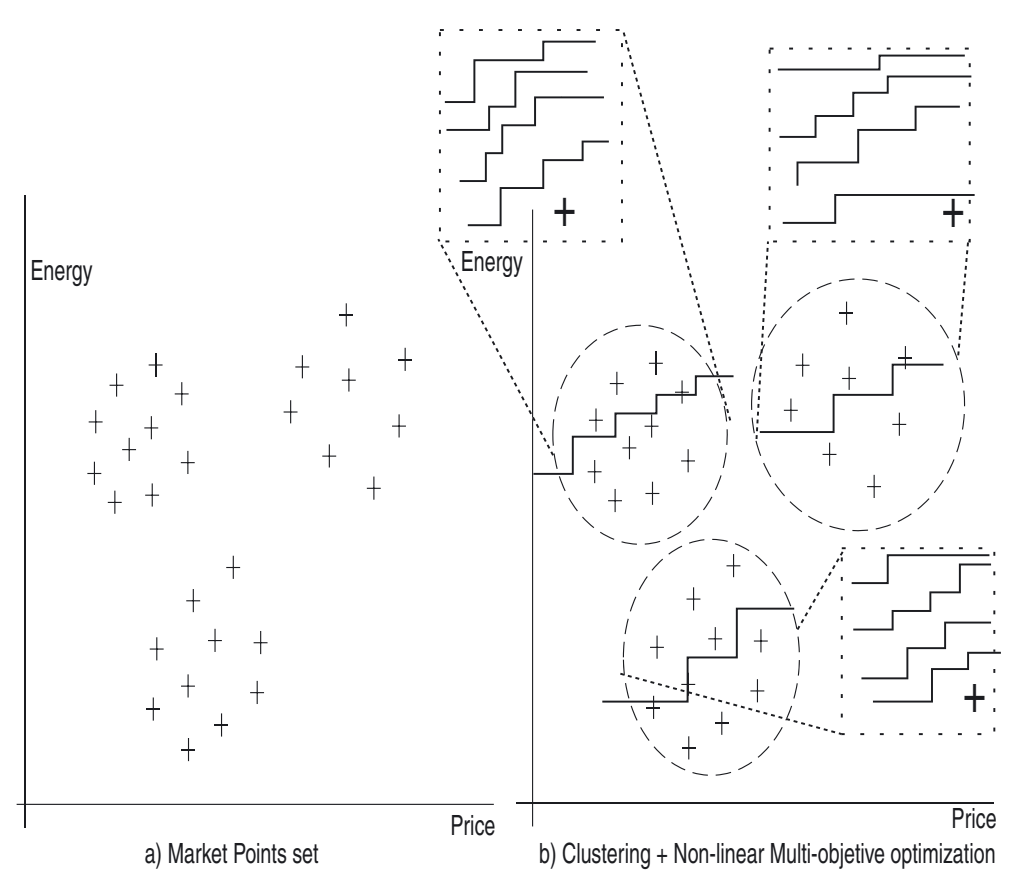

Figure 5. Approximation by classical methods: Clustering + Non-linear multi-objective optimization.

\subsection{Semi-Synthesized Problem Description}

The problem proposed here is more complex than the demonstration scenario shown in Section 3. Market points have been distributed resembling market points in a specific real situation (see Fig. 6(a) for actual data points for two days in the year 2000). The real problem in Fig. 6(a) has been simplified to 6(b). The reasons that forced us to use semi-synthetic data are:

- Supply curves: we have assumed a small number of aggregate supply curves exists, and a simple behavior for every firm (i.e., the supply curve only depends on the market segment given by the classifier). This is
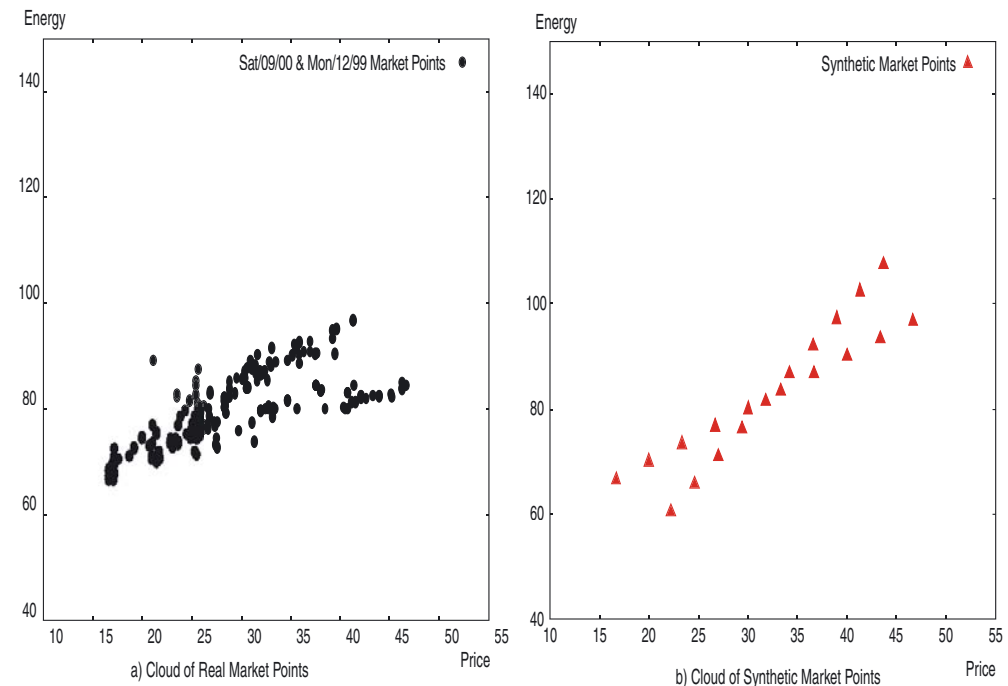

Figure 6. Saturday (September 2000) and Sunday (December 1999) market points (a) compared to semi-synthetic data (b). 


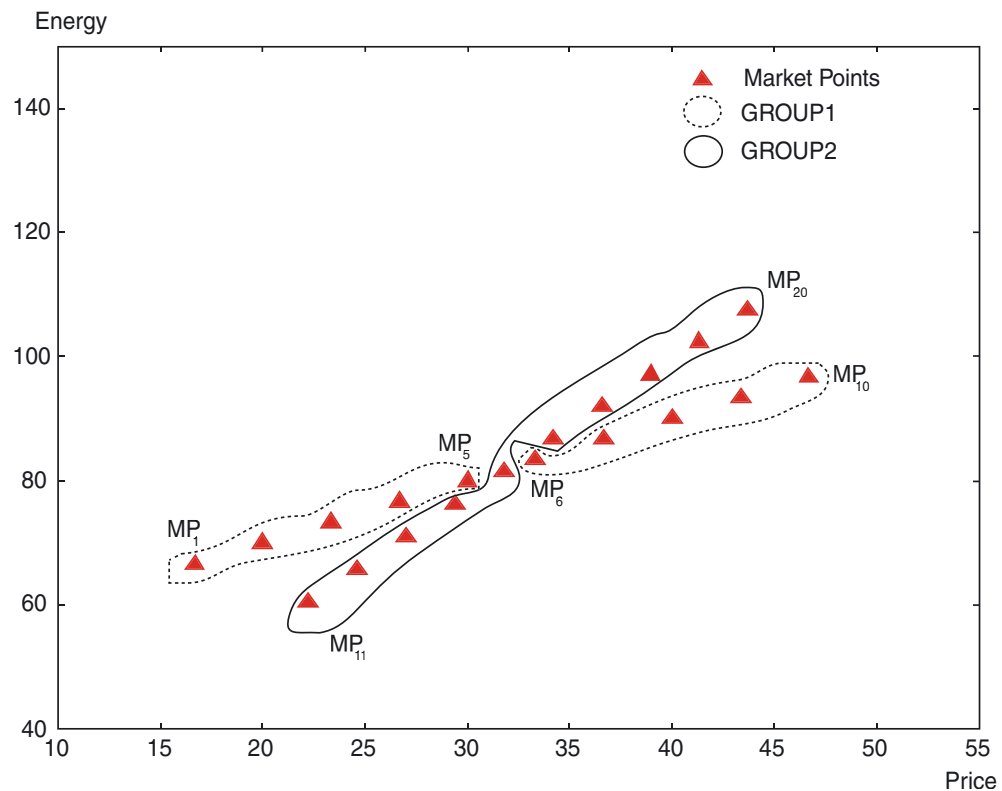

Figure 7. Cross-shaped cloud of market points for the semi-synthetic problem and the two clusters that should be obtained.

a simplification of the real case. In practice, each generator operates with certain autonomy, thus the actual aggregate supply curves are never exactly the same in two different markets. By replacing them with semi-synthetic curves, we can know whether the deviations between desired add real output of our algorithm are due to the genetic algorithm, or they are inherent in the dispersion of the data.

- Demand elasticity: The steepness of the demand curves was not provided by the MO. Therefore, we assume a plausible value.

- Both the demand and the cost functions are linearizations of the actual ones.

We decided to generate 20 points in two clusters, assuming that all points belong to weekends in the same season (and therefore the segmentation depends only on the hour of the day, see Fig. 7). We wish to recover the four individual supply curves (the Spanish electrical market is made up of four major generating agents) that originated the situation in Fig. 6(b).

The input data includes:

1. 20 market points, $M P_{i}, i=1, \ldots 20$ (pairs pricequantity)

2. 20 Demand functions, which were assigned the same elasticity (-2). For instance, demand function for the market point characterized by the pair (price $=16.67$, quantity $=66.67$ ) is $-2 *$ price +100 .

3. 4 linear production cost functions, (one for each firm) which are

$$
\begin{aligned}
& C_{0}(q)=8 q+10 \\
& C_{1}(q)=10 q+20 \\
& C_{2}(q)=12 q+30 \\
& C_{3}(q)=11 q+25
\end{aligned}
$$

4. The quotient between unitary profits of all firms (1, in this case).

5. The number of prototypes (2).

The prototypes used to generate the market points (the desired output of the process) are shown in Table 4.

\subsection{Experimental Framework}

5.2.1. Crossover Operation. The first decision that must be made in CGM algorithm concerns the method for selecting prototypes in the crossover operation. We have evaluated two alternatives: only 1 prototype, selected at random, is interchanged between the individuals being crossed ("coevol-1-random") or all prototypes are used in a kind of uniform crossover 
Table 4. Objective individual strategies for the cross shaped problem. Prototypes are piecewise linear, with 3 segments. Each compound strategy comprises 2 prototypes, and the classifier system has two rules.

\begin{tabular}{ll}
\hline & $M P_{1-10}$ \\
E0 & $(16.67,2.13)(26.67,2.17)(36.67,2.21)(46.67,2.25)$ \\
E1 & $(16.67,7.39)(26.67,7.69)(36.67,7.99)(46.67,8.29)$ \\
E2 & $(16.67,42.52)(26.67,51.02)(36.67,59.52)(46.67,68.02)$ \\
E3 & $(16.67,14.55)(26.67,15.65)(36.67,16.75)(46.67,17.85)$ \\
& $M P_{11-20}$ \\
E0 & $(16.67,1.93)(26.67,2.08)(36.67,2.23)(46.67,2.38)$ \\
E1 & $(16.67,6.40)(26.67,7.33)(36.67,8.26)(46.67,9.19)$ \\
E2 & $(16.67,29.35)(26.67,46.45)(36.67,63.55)(46.67,80.65)$ \\
E3 & $(16.67,10.63)(26.67,14.33)(36.67,18.03)(46.67,21.73)$ \\
\hline
\end{tabular}

Table 5. Framework to compare GM and CGM. Since the crossover is very disruptive, the mutation operator did not improve the convergence and it was not used. For the same reason, the probability of crossover has to be lowered as the search advances.

\begin{tabular}{ll}
\hline Selection type: & Tournament \\
Tournament size: & 4 \\
Number of pops.: & 4 populations in coevolutionary model \\
Size of pops.: & $100-1000$ \\
Iterations: & $100-500$ \\
Crossover probability: & Linear descending from $100.0 \%$ \\
Mutation probability: & $0.0 \%$
\end{tabular}

that we have labelled "coevol-all" (see Fig. 8). The framework for this and future genetic experiments in this section is shown in Table 5. GM crossover is different because individuals do not codify the strategies of one firm but a combination comprising a set of strategies for every firm. CGM crossover can be extended to this schema: evol-1-random se- lects one firm's strategies from both parents and applies "coevol-1-random" crossover to them. "Evol-all" consists of applying "coevol-1-random" to all firms (see Fig. 9).

Observe that the settings for some of these operators are not the usual ones: mutation did not improve the convergence speed so it was not used (see Section A.7). Our experiments have also shown that the crossover was very disruptive, even after the modifications proposed in Section 2.3 were applied. Notice that the probability of crossover is lowered as the search advances due to the same reason (see Section A.6).

5.2.2. Fitness Function. Regular fitness calculation of a GM individual consists of making the firms compete with each other. This will be called "intra-i" fitness. An alternative fitness involves the competition of each firm with firms from another individual selected at random from the population. This fitness will labelled "extra-i" (see Fig. 10).

5.2.3. Summary of the Decisions Made. The diagram in Fig. 11 shows the decisions made to obtain the final genetic configuration. The first decision consists in deciding between multi-objective and scalar fitness, using CGM to evaluate both fitnesses. Our experiments (see Section A.2) conclude that multi-objective fitness is more effective than scalar fitness. Therefore, scalar fitness will not be used in the remaining decisions.

Again, in CGM, we have to choose one crossover operator between "coevol-1-random" and "coevol-all." Results in Section A.3 state that "coevol-1-random" is the best one. Therefore, "coevol-1-random" is selected as the choice crossover operator for individuals in CGM for all tests.

Two crossover operators for GM individuals are analysed: "evol-1-random" and "evol-all." In this case,

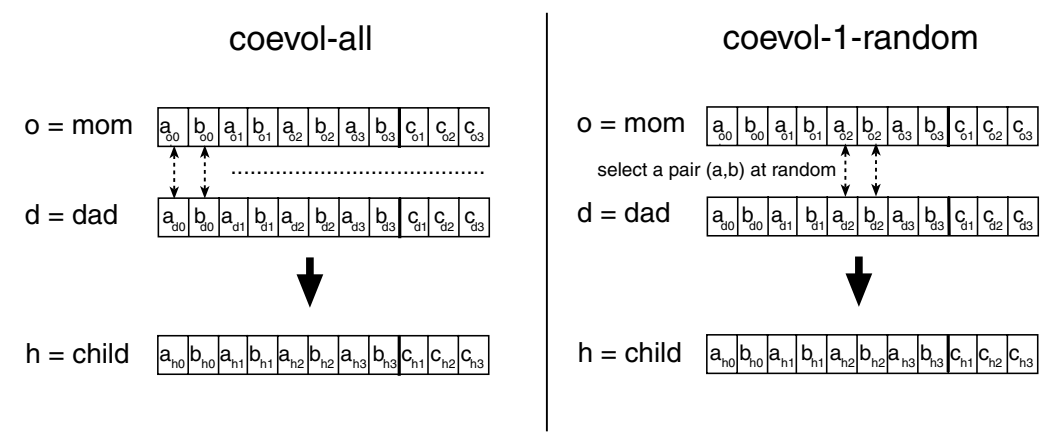

Figure 8. Types of crossover operators for CGM, (coevol-all and coevol-1-random). 


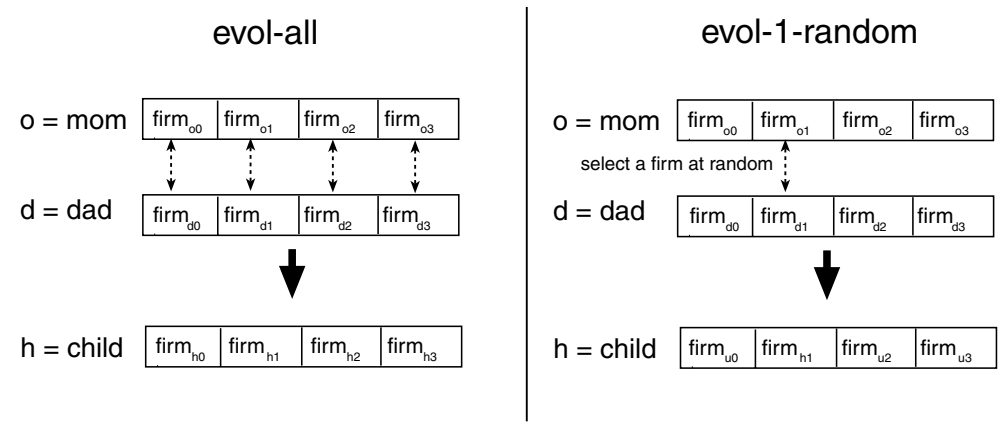

Figure 9. Types of crossover operators for GM, (evol-all and evol-1-random).

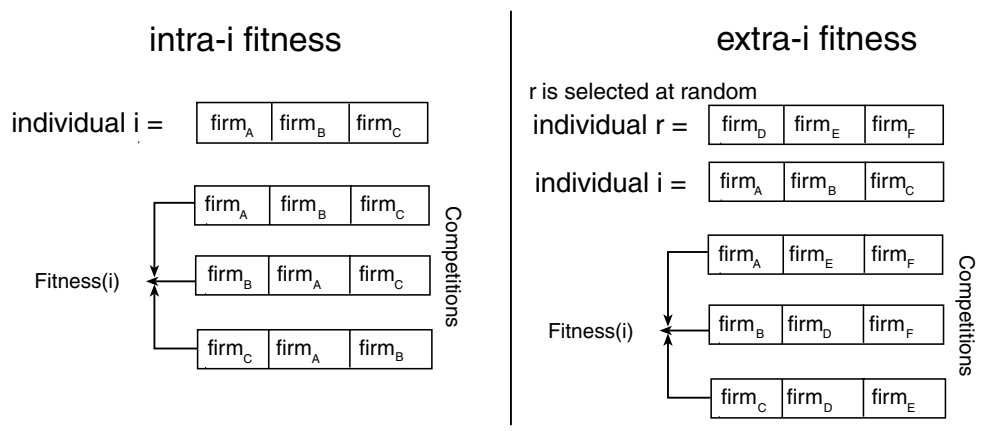

Figure 10. Types of fitness functions for GM, (intra-i and extra-i).

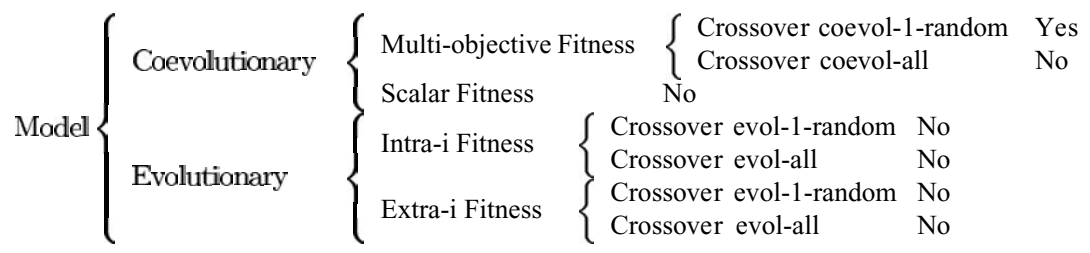

Figure 11. Diagram with the decisions taken to select fitness functions and crossover operators for GM and CGM.

results from Section A.5 show us that both crossover operators have a similar behavior.

"Extra-i" and "intra-i" fitness for GM have been studied, too. The results (see experiments in Section A.4) demonstrate that "extra-i" fitness is better than "intra-i" fitness in most of the cases studied.

Finally, after comparing GM and CGM results (see Section A.8), we can conclude that CGM with multiobjective fitness and the "coevol-1-random" crossover operator is more effective than GM.

\subsection{Comparison Between KGM and CGM}

We have selected the best model obtained in the previous section, CGM, to compare it with an alternative method based on "a priori" clustering (see Section 4). KGM takes two steps:

1. Clustering of market points using the K-means algorithm.

2. For each cluster of market points from the preceding step, CGM without the classification system (one prototype) is applied to fit one polygonal curve to the market points of every cluster.

After Section A.8 results, we can conclude that CGM is rather more effective than GM and KGM, but KGM can compete with the non-coevolutionary solution GM (see Table 14 in Appendix A.8). Therefore, the choice method is now CGM with coevol-1-random crossover operator and multi-objective fitness. 


\subsection{Analysis of Results and Applications of the Method}

We will show the practical relevance of this algorithm by using it to solve a practical problem. Suppose we wish to predict what would happen if one firm increases its price.

One would expect that the total amount of total energy sold in the pool decreases, and, therefore, the final price increases. However, the share of the firms will change, too, and it is possible that the increase is profitable for the enterprise that issued it.

Let us assume the change is small enough so the remaining enterprises do not react and alter their respective strategies. In case this is not true, the tool we have developed here is not valid. One should run an algorithm similar to this in structure, but with a change in the fitness function. In order to simulate the reaction of the enterprises, it is no longer true that the strategies match the historical data, and the fitness must depend mainly on the profit of each enterprise, thus the genetic algorithm can evolve towards the optimal strategy definition in terms of the game theory. This point will be recalled in Section 6, "Concluding Remarks and Future Work".

After our algorithm has been used to extract the individual supply curves, we follow the next schema:

1. A simulation of the pool is run to calculate the profit of all firms.

2. The strategy of one firm is altered.

3. A second simulation is run to calculate the new profit.

5.4.1. Experimental Framework. Tests with an estimated pool from each model studied (GM, CGM and $n$-KGM models, where $n$ is the number of clusters) have been made. Let us suppose first that we are firm 0 , the firm with the lowest market share. We will increase our supply curve by $1 \%$ and evaluate the new profits. Then the supply is increased by $5 \%$ and the analysis repeated. Finally, a second, identical experiment will be carried out, but from the point of view of firm 2 , the biggest company.

The value Perc_error measures the error that CGM, GM and KGM introduce into this process:

Perc_error $=100$

$\mid$ Profit increase(estimated pool) - Profit increase(actual pool)| Profit increase (actual pool)
It can be observed that the coevolutionary method presents (see Table 6) the lowest maximum percentage error (CGM, $5.05 \%$ of maximum percentage error) of all the methods studied. 1-KGM is the second, with $21.25 \%$ of error, after GM with $38.33 \%$ and last 2-KGM. These results suggest that clustering (KGM) is not effective. Besides the adjustment to the market points is better when the number of 2 clusters is increased (see Table 14 in Appendix A.8), this does not imply that the resulting pool is nearer to the actual one. This is experimentally stated in Table 6, where 2-KGM method obtains an error of $83.45 \%$ as opposed to $21.25 \%$ in the $1-\mathrm{KGM}$ method. Therefore, we can conclude that the coevolutionary analysis of markets is a more precise technique than both non coevolutionary analysis and ordinary segmentation with submodel fitting for this application.

\section{Concluding Remarks and Future Work}

Coevolutionary genetic models are usually used to simulate natural systems with multiple agents of independent behavior, where mathematical models are too complex to be applied. Here, we have experimentally shown that a coevolutionary genetic model is more effective than a regular genetic model or a $K$-means algorithm plus genetic algorithm based on non-linear regression. On the other hand, it has been stated that multi-objective fitness is also more effective than real value fitness. Multi-objective fitness allows goals to be explored in parallel, avoiding mutual obstruction, and, therefore, the premature convergence to a local optimal.

From the point of view of a firm that manages our estimated pool, this method shows the influence of a slight variation in its strategy over its profits, given a certain market situation. This way, a firm can adjust its strategy and improve its profits.

The following step to improve this algorithm consists in designing a tool capable of generating the optimal pool, given the demand and the production costs of the firms. From the Market Operator's point of view, it is useful to know the theoretical optimal strategies for a set of market points in a perfect oligopolistic situation, which is a non-linear extension to the Cournot problem [20]. This information will serve to estimate the difference between the real profit of the pool and the theoretical maximum profit if competition is perfect, therefore detecting illegal agreements between 


\begin{tabular}{|c|c|c|c|c|c|c|}
\hline Firm & $\%$ Modif. & Method & Prof $f_{\text {before }}$ & Prof $_{\text {after }}$ & Increase & Percentage error \\
\hline 0 & 1.0 & Actual & 883.2 & 892.0 & +8.8 & 0.00 \\
\hline 0 & 1.0 & CGM & 883.1 & 891.9 & +8.8 & 0.00 \\
\hline 0 & 1.0 & $\mathrm{GM}$ & 881.2 & 890.1 & +8.8 & 0.79 \\
\hline 0 & 1.0 & 1-KGM & 886.3 & 895.1 & +8.8 & 0.09 \\
\hline 0 & 1.0 & 2-KGM & 864.2 & 872.5 & +8.4 & 5.24 \\
\hline 0 & 5.0 & Actual & 883.2 & 927.3 & +44.1 & 0.00 \\
\hline 0 & 5.0 & CGM & 883.2 & 927.2 & +44.1 & 0.01 \\
\hline 0 & 5.0 & $\mathrm{GM}$ & 881.2 & 925.7 & +44.4 & 0.81 \\
\hline 0 & 5.0 & 1-KGM & 886.3 & 930.4 & +44.0 & 0.10 \\
\hline 0 & 5.0 & 2-KGM & 864.2 & 906.0 & +41.8 & 5.26 \\
\hline 2 & 1.0 & Actual & 23887.3 & 23899.6 & +12.3 & 0.00 \\
\hline 2 & 1.0 & CGM & 23929.2 & 23942.0 & +12.8 & 4.10 \\
\hline 2 & 1.0 & $\mathrm{GM}$ & 23593.6 & 23601.3 & +7.7 & 37.40 \\
\hline 2 & 1.0 & 1-KGM & 23922.9 & 23932.7 & +9.8 & 20.33 \\
\hline 2 & 1.0 & 2-KGM & 23893.6 & 23915.5 & +21.9 & 78.05 \\
\hline 2 & 5.0 & Actual & 23887.3 & 23944.7 & +57.4 & 0.00 \\
\hline 2 & 5.0 & CGM & 23989.5 & 23929.2 & +60.3 & 5.05 \\
\hline 2 & 5.0 & $\mathrm{GM}$ & 23593.6 & 23629.0 & +35.4 & 38.33 \\
\hline 2 & 5.0 & 1-KGM & 23922.9 & 23968.1 & +45.2 & 21.25 \\
\hline 2 & 5.0 & 2-KGM & 23893.6 & 23998.9 & +105.3 & 83.45 \\
\hline
\end{tabular}

generators. In addition, it will serve to simulate the pool under large changes in the supply curves of one firm, overpassing the limitations of the analysis in the last section.

\section{Appendix A: Experiments}

This Appendix includes numerical results for experiments made with the semi-synthetic problem from Section 5 to select the best genetic parameters. These results have been moved here to improve the readability of the paper. General framework and specific error functions are described in Section A.1.

Section A. 2 shows results for multi-objective fitness compared to scalar fitness using CGM. Results of comparing proposed crossover operators for CGM are included in Section A.3. Different fitness function results for GM are shown in Section A.4. Section A.5 retrieves results for crossover operators with GM. Studies of the crossover and mutation probability are presented in Sections A.6 and A.7 respectively and finally a summary table is included in Section A.8.

\section{A.1. Framework and Error Measurement}

All tests were made using genetic parameters from Table 5. Different fitness functions and crossover operators will be analyzed in each of the following sections.

In order to provide complete information about the results obtained, two error functions have been used to measure the distance between the estimated curves and the original supply curves:

- Error ${ }_{1}$ measures the mean percentage error of estimated quantity for all firms in each market.

- Error 2 measures the mean percentage error of estimated energy share in each market for all firms.

\section{A.2. Mono-Objective vs. Multi-Objective Fitness, Coevolutionary Model}

Tests made with multi-objective fitness, scalar fitness and a coevol-1-random crossover operator (see results in Section A.3) using CGM are in Table 7. Results 
Table 7. Comparison of errors using multi-objective and scalar fitness for CGM.

\begin{tabular}{lcccccc}
\hline Fitness & Pop & Iter & Error & $\sigma_{\text {error }_{1}}$ & Error $_{2}$ & $\sigma_{\text {error }_{2}}$ \\
\hline Multi-objective fitness & 100 & 200 & $\mathbf{2 . 4 2 7}$ & $\mathbf{0 . 8 7 2}$ & $\mathbf{0 . 4 1 1}$ & $\mathbf{0 . 1 5 7}$ \\
Scalar fitness & 100 & 200 & 253.896 & 126.628 & 22.695 & 6.821 \\
Multi-objective fitness & 200 & 200 & $\mathbf{2 . 1 8 9}$ & $\mathbf{0 . 9 6 5}$ & $\mathbf{0 . 4 0 0}$ & $\mathbf{0 . 2 1 3}$ \\
Scalar fitness & 200 & 200 & 240.562 & 99.271 & 20.013 & 6.778 \\
Multi-objective fitness & 300 & 200 & $\mathbf{1 . 8 3 5}$ & $\mathbf{0 . 6 9 1}$ & $\mathbf{0 . 3 4 2}$ & $\mathbf{0 . 1 3 1}$ \\
Scalar fitness & 300 & 200 & 261.687 & 64.373 & 19.510 & 4.912 \\
Multi-objective fitness & 400 & 200 & $\mathbf{1 . 8 4 2}$ & $\mathbf{0 . 6 6 3}$ & $\mathbf{0 . 3 6 4}$ & $\mathbf{0 . 1 4 9}$ \\
Scalar fitness & 400 & 200 & 269.891 & 72.581 & 22.769 & 4.962 \\
Multi-objective fitness & 500 & 200 & $\mathbf{1 . 6 3 1}$ & $\mathbf{0 . 7 4 8}$ & $\mathbf{0 . 3 3 2}$ & $\mathbf{0 . 1 7 6}$ \\
Scalar fitness & 500 & 200 & 246.641 & 96.271 & 20.926 & 5.624 \\
Multi-objective fitness & 1000 & 200 & $\mathbf{1 . 1 1 8}$ & $\mathbf{0 . 5 2 3}$ & $\mathbf{0 . 1 9 7}$ & $\mathbf{0 . 0 8 8}$ \\
Scalar fitness & 1000 & 200 & 255.751 & 62.520 & 21.408 & 4.483 \\
Multi-objective fitness & 500 & 500 & $\mathbf{1 . 3 2 7}$ & $\mathbf{0 . 7 8 2}$ & $\mathbf{0 . 2 7 0}$ & $\mathbf{0 . 1 8 1}$ \\
Scalar fitness & 500 & 500 & 215.066 & 112.140 & 17.916 & 6.920 \\
Multi-objective fitness & 1000 & 500 & $\mathbf{1 . 1 6 8}$ & $\mathbf{0 . 6 4 0}$ & $\mathbf{0 . 2 5 3}$ & $\mathbf{0 . 1 3 9}$ \\
Scalar fitness & 1000 & 500 & 223.648 & 126.607 & 19.170 & 8.001 \\
\hline & & & & & & \\
\hline
\end{tabular}

are the average of 10 runs with each set of parameters: type of fitness (Fitness), number of individuals by population (Pop) and number of iterations (Iter).

Multi-objective fitness obtains the best solution with both quality criteria (absolute error - Error $_{1}$ - and distribution of market shares -Error $2-$ ). It seems that multiobjective fitness converges in parallel to a global minimum in its two components (variance of unitary profits -Fitness $b_{b}$ - and square error -Fitness ${ }_{a}-$ ) while scalar fitness, which is a weighted sum of both, is dragged by the easiest convergence component, causing convergence to a local minimum (see Fig. 12).

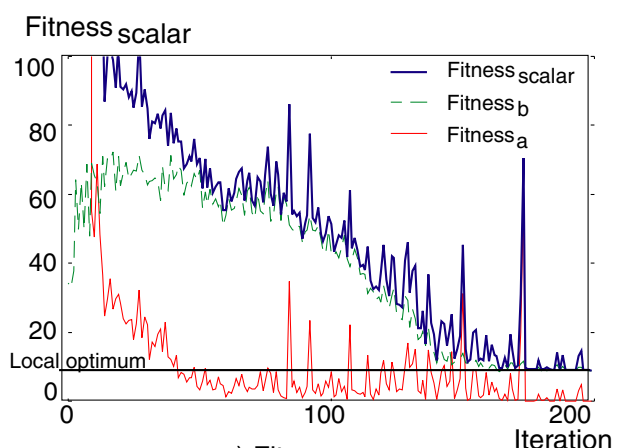

a) Fitness scalar

\section{A.3. Selection of the Crossover Operation, Coevolutionary Model}

Numerical results for the two proposed crossover operators, coevol-1-random and coevol-all, and multiobjective fitness are in Table 8 . It shows average results of 10 runs with each set of parameters: type of crossover operator (Crossover), number of individuals by population (Pop) and number of iterations (Iter).

Although the margin between both variants is small, coevol-1-random is better than coevol-all in all tests.

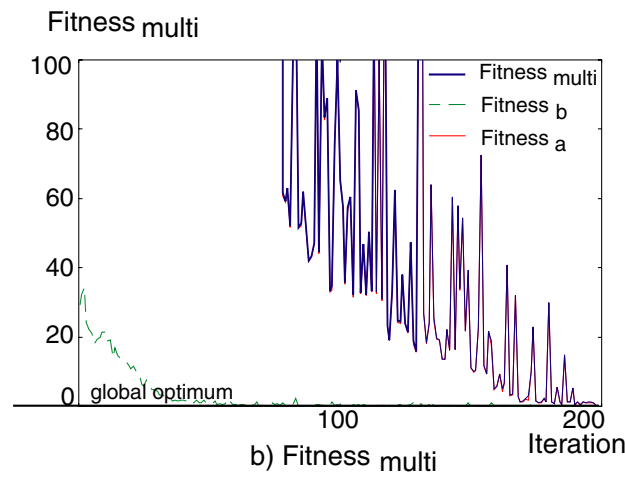

Figure 12. Comparison of Scalar (a) vs. Multi-objective (b) Fitness Progression. 
Table 8. Crossover methods comparison for a coevolutionary model.

\begin{tabular}{lrrrrrr}
\hline Crossover & Pop & Iter & Error $_{1}$ & $\sigma_{\text {Error }_{1}}$ & Error $_{2}$ & $\sigma_{\text {Error }_{2}}$ \\
\hline coevol-all & 500 & 200 & 2.310 & 0.997 & 0.383 & 0.182 \\
coevol-1-random & 500 & 200 & $\mathbf{1 . 6 3 1}$ & $\mathbf{0 . 7 4 8}$ & $\mathbf{0 . 3 3 2}$ & $\mathbf{0 . 1 7 6}$ \\
coevol-all & 500 & 500 & 1.744 & 0.755 & 0.356 & 0.167 \\
coevol-1-random & 500 & 500 & $\mathbf{1 . 3 2 7}$ & $\mathbf{0 . 7 8 2}$ & $\mathbf{0 . 2 7 0}$ & $\mathbf{0 . 1 8 1}$ \\
coevol-all & 1000 & 200 & 1.778 & 0.526 & 0.282 & 0.073 \\
coevol-1-random & 1000 & 200 & $\mathbf{1 . 1 1 8}$ & $\mathbf{0 . 5 2 3}$ & $\mathbf{0 . 1 9 7}$ & $\mathbf{0 . 0 8 8}$ \\
coevol-all & 1000 & 500 & 1.241 & 0.715 & 0.246 & 0.159 \\
coevol-1-random & 1000 & 500 & $\mathbf{1 . 1 6 8}$ & $\mathbf{0 . 6 4 0}$ & $\mathbf{0 . 2 5 3}$ & $\mathbf{0 . 1 3 9}$
\end{tabular}

\section{A.4. Fitness Function, Non Coevolutionary Model}

Both GM fitness functions, "intra-i" fitness and "extrai," fitness have been tested with both GM crossover operators, evol-1-random and evol-all (see Section A.5).

We propose an alternative to regular fitness (intra-i fitness) that consists in making each firm comprising an individual compete with firms from another individual selected at random from the population. Table 9 contains average results of 10 runs with each set of parameters: type of fitness (Fitness), type of crossover operator, number of individuals by population (Pop) and number of iterations (Iter).

Table 9. Intra-i fitness vs. extra-i fitness for GM.

\begin{tabular}{lrrrrrr}
\hline Fitness & Pop & Iter & Error $_{1}$ & $\sigma_{\text {error }}$ & Error & $\sigma_{\text {error }}$ \\
\hline intra-i & 500 & 200 & 12.742 & 4.032 & 2.245 & 0.885 \\
extra-i & 500 & 200 & 7.844 & 1.744 & 0.793 & 0.333 \\
intra-i & 500 & 500 & 5.708 & 1.880 & 1.252 & 0.443 \\
extra-i & 500 & 500 & 4.495 & 1.959 & 0.718 & 0.252 \\
intra-i & 1000 & 200 & 11.181 & 2.660 & 1.899 & 0.503 \\
extra-i & 1000 & 200 & 7.230 & 1.276 & 0.720 & 0.346 \\
intra-i & 1000 & 500 & 4.822 & 1.163 & 1.003 & 0.237 \\
extra-i & 1000 & 500 & 4.223 & 1.858 & 0.607 & 0.220 \\
& & Evol-1-random crossover & & \\
intra-i & 500 & 200 & 35.972 & 17.795 & 4.131 & 2.317 \\
extra-i & 500 & 200 & 27.063 & 15.330 & 1.463 & 0.495 \\
intra-i & 500 & 500 & 4.268 & 1.746 & 0.899 & 0.410 \\
extra-i & 500 & 500 & 5.266 & 3.894 & 0.962 & 0.613 \\
intra-i & 1000 & 200 & 14.577 & 5.724 & 1.888 & 0.690 \\
extra-i & 1000 & 200 & 17.239 & 9.279 & 1.208 & 0.654 \\
intra-i & 1000 & 500 & 3.949 & 1.331 & 0.867 & 0.370 \\
extra-i & 1000 & 500 & 3.875 & 1.871 & 0.750 & 0.340 \\
\hline & & & & & &
\end{tabular}

In conclusion, we can affirm that the method of competition of external fitness (extra-i) is better in almost all the cases studied. This difference is attenuated as the number of individuals and iterations grows.

\section{A.5. Crossover Operator, Non Coevolutionary Model}

We propose two GM crossover operators, one similar to coevol-1-random, but crossing whole firms and not prototype strategies. Let us define a CGM scenario with $p$ populations, $i$ individuals by population and crossover probability of $c$, so the expected next generation number of individuals produced by the crossing operator will be:

$$
\begin{aligned}
\text { Crossed CGM individuals } / \text { population } & =\frac{2 c i}{100+c} \\
\text { Total crossed CGM individuals } & =\frac{2 c i p}{100+c}
\end{aligned}
$$

The population of the equivalent GM has $i$ individuals (like a CGM population), but each GM individual will be made up of $p$ firms (a firm is a set of strategies, such as a CGM individual). According to this, if the crossing between two GM individuals composed by $m$ population segments is implemented as the crossing of all the corresponding firms comprising it (evol-all), the number of firms after crossing will be the same number as CGM individuals:

$$
\begin{aligned}
\text { Crossed GM firms/GM individual } & =\frac{2 c i}{100+c} \\
\text { Total crossed GM firms } & =\frac{2 c i p}{100+c}
\end{aligned}
$$

Thus, we propose a new GM crossover consisting of the random selection of only one firm for the crossing (evol-1-random), instead of crossing all the firms. The results of Table 10 are the average of 10 runs for each set of parameters: type of crossover (Crossover), number of individuals by population (Pop) and number of iterations (Iter).

Table 10 shows that evol-1-random crossover improves with the population size (Pop) and the number of iterations (Iter). Evol-1-random is better than evol-all from 500 iterations. This demonstrates that the number of crossings is not totally decisive in the problem that we are raising, since the errors (Error ${ }_{1}$, and Error $\left._{2}\right)$ are not improved with a new evolutionary crossover 
Table 10. Crossover methods comparison for evolutionary model.

\begin{tabular}{lcccccc}
\hline Crossover & Pop & Iter & Error $_{1}$ & $\sigma_{\text {error }_{1}}$ & Error $_{3}$ & $\sigma_{\text {error }_{3}}$ \\
\hline evol-all & 500 & 200 & 12.742 & 4.032 & 2.245 & 0.885 \\
evol-1-random & 500 & 200 & 35.972 & 17.795 & 4.131 & 2.317 \\
evol-all & 1000 & 200 & 11.181 & 2.660 & 1.899 & 0.503 \\
evol-1-random & 1000 & 200 & 14.577 & 5.724 & 1.888 & 0.690 \\
evol-all & 500 & 500 & 5.708 & 1.880 & 1.252 & 0.443 \\
evol-1-random & 500 & 500 & 4.268 & 1.746 & 0.899 & 0.410 \\
evol-all & 1000 & 500 & 4.822 & 1.163 & 1.003 & 0.237 \\
evol-1-random & 1000 & 500 & 3.949 & 1.331 & 0.867 & 0.370 \\
\hline
\end{tabular}

operator with respect to the equivalent coevolutionary model.

\section{A.6. Crossover Probability Study}

All the previous studies were carried out using a crossover probability calculated with the expression $100\left(1-\frac{\text { iter }}{\text { NTotalIter }}\right)$, with which the best practical results were obtained. To confirm this statement, the results were tabulated for several tests with a constant crossover probability (between $1.0 \%$ and 100\%) in the CGM model with multi-objective fitness, using the crossover operator "coevol-1-random" (see Table 11).

Observe that as the crossover probability increases, the value of Error $_{1}$ worsens, and the minimum crossover probability of $10 \%$ is obtained. This indi-

Table 11. Errors from applying a constant crossover probability of $10-100 \%$ to the CGM model combined with the multiobjective fitness and crossover operator "coevol-1-random".

\begin{tabular}{ccccc}
\hline \%Crossover probability & Error $_{1}$ & $\sigma_{\text {Error }_{1}}$ & Error $_{3}$ & $\sigma_{\text {error }_{3}}$ \\
\hline Descending Probability & $\mathbf{1 . 6 3 1}$ & $\mathbf{0 . 7 4 8}$ & $\mathbf{0 . 3 3 2}$ & $\mathbf{0 . 1 7 6}$ \\
1 & 5.340 & 2.957 & 0.595 & 0.257 \\
5 & 3.419 & 0.950 & 0.611 & 0.232 \\
10 & 3.010 & 1.344 & 0.554 & 0.204 \\
20 & 3.580 & 2.350 & 0.530 & 0.223 \\
30 & 3.374 & 1.501 & 0.594 & 0.292 \\
40 & 3.730 & 1.250 & 0.652 & 0.220 \\
50 & 4.341 & 1.569 & 0.770 & 0.309 \\
60 & 4.604 & 1.900 & 0.806 & 0.284 \\
70 & 6.418 & 3.029 & 1.043 & 0.460 \\
80 & 6.160 & 2.867 & 0.997 & 0.413 \\
90 & 6.099 & 2.659 & 0.971 & 0.406 \\
100 & 6.511 & 2.556 & 1.100 & 0.477 \\
\hline
\end{tabular}

Table 12. Comparison of the best results obtained with a constant crossover probability as opposed to a descending crossover probability with a linear function

\begin{tabular}{lcccc}
\hline Crossover probability & Error $_{1}$ & $\sigma_{\text {Error }_{1}}$ & Error $_{3}$ & $\sigma_{\text {error }_{3}}$ \\
\hline Const. 10\% & 3.010 & 1.344 & 0.554 & 0.204 \\
Const. 20\% & 3.580 & 2.350 & 0.530 & 0.223 \\
Descending Probability & $\mathbf{1 . 6 3 1}$ & $\mathbf{0 . 7 4 8}$ & $\mathbf{0 . 3 3 2}$ & $\mathbf{0 . 1 7 6}$ \\
\hline
\end{tabular}

cates that the population diversity remains high until the end of CGM learning, when the crossover probability is still high, which favors high dispersion. For this reason, we decided on the use of a descending crossover probability. The minimum errors, Error $_{1}$ as well as Error 2 , obtained with constant crossover probability, do not improve the results obtained with the descending probability of $1.631 \%$ (see Table 12 ).

\section{A.7. Mutation Probability Study}

Uniform arithmetic crossover operators already include a random factor, which is why the possibility of not using a mutation operator was assessed. To experimentally contrast this decision, testing was carried out with positive mutation probabilities from 1.0 to $10 \%$. Table 13 contains the results obtained with a mutation operator applied with positive probabilities ( $\%$ Mut.) and without it (probability $0 \%$ ), both combined with the CGM model with multi-objective fitness and crossover operator "coevol-1-random".

In view of the results obtained, we can say that the errors increase as the mutation probability increases, which is why it was decided not to use this operator.

\section{A.8. Summary}

As shown in Table 14, it can be concluded that the coevolutionary model using multi-objective fitness is

\begin{tabular}{|c|c|c|c|c|}
\hline$\%$ Mut. & Error $_{1}$ & $\sigma_{\text {Error }_{1}}$ & Error $_{3}$ & $\sigma_{\text {Error }_{3}}$ \\
\hline 0.0 & 1.631 & 0.748 & 0.332 & 0.176 \\
\hline 0.5 & 1.949 & 0.834 & 0.373 & 0.148 \\
\hline 1.0 & 2.323 & 1.286 & 0.420 & 0.183 \\
\hline 5.0 & 3.024 & 0.587 & 0.488 & 0.083 \\
\hline 10.0 & 3.132 & 0.330 & 0.450 & 0.073 \\
\hline
\end{tabular}


Table 14. Comparison of errors obtained with CGM, GM and KGM.

\begin{tabular}{llllllll}
\hline Model & \multicolumn{1}{c}{ Cross./fitness } & Pop & Iter & Error $_{1}$ & $\sigma_{\text {error }_{1}}$ & Error $_{2}$ & $\sigma_{\text {error }_{2}}$ \\
\hline 1-KGM & - & 500 & 200 & 3.716 & 0.000 & 0.423 & 0.000 \\
2-KGM & - & 500 & 200 & 3.580 & 0.000 & 0.515 & 0.000 \\
GM & All/extra-i fitness & 500 & 200 & 7.844 & 1.744 & 0.793 & 0.333 \\
CGM & 1-random/multi-objective fitness & 500 & 200 & $\mathbf{1 . 6 3 1}$ & $\mathbf{0 . 7 4 8}$ & $\mathbf{0 . 3 3 2}$ & $\mathbf{0 . 1 7 6}$ \\
GM & 1-random/intra-i fitness & 500 & 500 & 4.268 & 1.746 & 0.899 & 0.410 \\
CGM & 1-random/multi-objective fitness & 500 & 500 & $\mathbf{1 . 3 2 7}$ & $\mathbf{0 . 7 8 2}$ & $\mathbf{0 . 2 7 0}$ & $\mathbf{0 . 1 8 1}$ \\
GM & All/extra-i fitness & 1000 & 200 & 7.230 & 1.276 & 0.720 & 0.346 \\
CGM & 1-random/multi-objective fitness & 1000 & 200 & $\mathbf{1 . 1 1 8}$ & $\mathbf{0 . 5 2 3}$ & $\mathbf{0 . 1 9 7}$ & $\mathbf{0 . 0 8 8}$ \\
GM & 1-random/extra-i fitness & 1000 & 500 & 3.875 & 1.871 & 0.750 & 0.340 \\
CGM & 1-random/multi-objective fitness & 1000 & 500 & $\mathbf{1 . 1 6 8}$ & $\mathbf{0 . 6 4 0}$ & $\mathbf{0 . 2 5 3}$ & $\mathbf{0 . 1 3 9}$ \\
\hline
\end{tabular}

more effective than the evolutionary equivalent model, and also better than the equivalent KGM.

\section{Acknowledgments}

This research has been partly funded by the "Fundación Banco Herrero 2002 Research Grant" and by "Ministerio de Ciencia y Tecnología," under the research proyect TIC2002-04036-C05-05.

\section{References}

1. Ministerio de Industria y Energía, "Protocolo para el establecimiento de una nueva regulación del sistema eléctrico nacional," Technical report, Ministerio de Industria y Energía, 1996.

2. J. Arifovic, "Genetic algorithm learning and the cobweb model," Journal of Economic Dynamics and Control, vol. 18, pp. 3-28, 1994.

3. T.C. Price, "Using co-evolutionary programming to simulate strategic behaviour in markets," Journal of Evolutionary Economics, vol. 7, pp. 219-234, 1997.

4. R. Franke, "Coevolution and stable adjustments in the cobweb model," Journal of Evolutionary Economics, vol. 8, pp. 383406, 1998.

5. H. Dawid, Adaptative Learning by Genetic Algorithms, Springer-Verlag: Berlin, 1999.

6. W.W. Leontief, "Verzögerte angebotsanpassung und partielles gleinchgewicht," Zeitschrift für Nationalökonomie, vol. 5, pp. 670-676, 1934.

7. R.E. Marks, "Breeding hybrid strategies: Optimal behaviour for oligopolists," Journal of Evolutionary Economics, vol. 2, pp. 1738, 1992.

8. R.E. Marks and L.G. Cooper, "The complexity of competitive marketing strategies," Complexity International, vol. 6, 1999.

9. W.D. Hillis, "Co-evolving parasites improve simulated evolution as an optimization technique," Artificial Life II, pp. 41-47, 1991.
10. M.A. Potterm, K.A. De Jong, and J.J. Grefenstette, "A coevolutionary approach to learning sequential decision rules," in Proceedings of the Sixth International Conference on Genetic Algorithms, edited by Larry Eshelman, Morgan Kaufmann: San Francisco, CA, 1995, pp. 366-372.

11. J. Paredis, "Coevolutionary computation," Artificial Life, vol. 2, no. 4, pp. 355-375, 1995.

12. C.D. Rosin and R.K. Belew, "New methods for competitive coevolution," Evolutionary Computation, vol. 5, no. 1, pp. 1-29, 1997.

13. W.M. Spears and K.A. De Jong, "On the virtues of parameterized uniform crossover," in Proceedings of the Fourth International Conference on Genetic Algorithms, edited by R. Belew and L. Booker, Morgan Kaufman: San Mateo, CA, 1991, pp. 230236.

14. G. Syswerda, "Uniform crossover in genetic algorithms," in Proceedings of the Third International Conference on Genetic Algorithms, edited by R. Belew and L. Booker, Morgan Kaufman: San Mateo, CA, 1989, pp. 2-9.

15. Y.J. Lai and C.L. Hwang, Fuzzy Multiple Objective DecisionMaking: Methods and Applications, Springer-Verlag: New York, 1996.

16. D.A. Veldhuizen and G.B. Lamont, "Multiobjective evolutionary algorithms: Analyzing the state-of-the-art," Evolutionary Computation, vol. 8, no. 2, pp. 125-147, 2000.

17. C.M. Fonseca and P.J. Fleming, "An overview of evolutionary algorithms in multiobjective optimization," Evolutionary Computation, vol. 3, no. 1, pp. 1-16, 1995.

18. C.M. Fonseca and P.J. Fleming, "Multiobjective optimization and multiple constraint handling with evolutionary algorithmsPart II: A application example," IEEE Transactions on Systems, Man, and Cybernetics, Part A: Systems and Humans, vol. 28, no. 1 , pp. $38-47,1998$

19. J. Horn, N. Nafpliotis, and D.E. Goldberg, "A Niched Pareto genetic algorithm for multiobjective optimization," in Proceedings of the First IEEE Conference on Evolutionary Computation, IEEE World Congress on Computational Intelligence, IEEE Service Center: Piscataway, NJ, vol. 1, 1994, pp. 82-87.

20. A. Cournot, Recherches sur les Principies Mathématiques de la Théorie des Richesses, Hachette, Paris, 1838. 